\title{
Fluctuations in milk yield are heritable and can be used as a resilience indicator to breed healthy cows
}

\author{
G. G. Elgersma, ${ }^{*}$ G. de Jong, $\dagger$ R. van der Linde, $\dagger$ and H. A. Mulder* ${ }^{* 1}$ \\ *Wageningen University \& Research Animal Breeding and Genomics, PO Box 338, $6700 \mathrm{AH}$ Wageningen, the Netherlands \\ †CRV BV, Wassenaarweg 20, 6843 NW Arnhem, the Netherlands
}

\begin{abstract}
Automatic milking systems record an enormous amount of data on milk yield and the cow itself. These type of big data are expected to contain indicators for health and resilience of cows. In this study, the aim was to define and estimate heritabilities for traits related with fluctuations in daily milk yield and to estimate genetic correlations with existing functional traits, such as udder health, fertility, claw health, ketosis, and longevity. We used daily milk yield records from automatic milking systems of 67,025 lactations in the first parity from 498 herds in the Netherlands. We defined 3 traits related to the number of drops in milk yield using Student $t$-tests based on either a rolling average (drop rolling average) or a regression (drop regression) and the natural logarithm of the within-cow variance of milk yield (LnVar). Average milk yield was added to investigate the relationships between milk yield and these new traits. ASReml was used to estimate heritabilities, breeding values (EBV), and genetic correlations among these new traits and average milk yield. Approximate genetic correlations were calculated using correlations between EBV of the new traits and existing EBV for health and functional traits correcting for nonunity reliabilities using the Calo method. Partial genetic correlations controlling for persistency and average milk yield and relative contributions to reliability were calculated to investigate whether the new traits add new information to predict fertility, health, and longevity. Heritabilities were 0.08 for drop rolling average, 0.06 for drop regression, and 0.10 for LnVar. Approximate genetic correlations between the new traits and the existing health traits differed quite a bit, with the strongest correlations $(-0.29$ to -0.52$)$ between LnVar and udder health, ketosis, persistency, and longevity. This study shows that fluctuations in daily milk yield are heritable and that the variance of milk production is best among
\end{abstract}

Received June 1, 2017.

Accepted October 2, 2017.

${ }^{1}$ Corresponding author: Han.mulder@wur.nl the 3 fluctuations traits tested to predict udder health, ketosis, and longevity. Using the residual variance of milk production instead of the raw variance is expected to further improve the trait to breed healthy, resilient, and long-lasting dairy cows.

Key words: fluctuation, resilience, health, variance, big data

\section{INTRODUCTION}

The world of dairy farming is changing; the number of farms is decreasing, whereas the number of dairy cows per farm is increasing. Therefore, an easy-to-manage herd becomes more important. For a farmer, it is easier to manage the herd when the animals are healthier. A healthy herd has fewer cases of diseases, which are costly and time-consuming for a farmer; furthermore, fewer cases of diseases would increase feed efficiency, which would help to feed the growing world population.

In dairy cattle, many diseases, such as milk fever, mastitis, ketosis, and lameness, often cause a decrease in milk yield (Rajala-Schultz et al., 1999; Windig et al., 2005; Onyiro et al., 2008). The pattern of the drop in milk yield differs. Milk fever can affect milk yield of cows for up to $6 \mathrm{wk}$, with a loss in milk production of 1.1 to $2.9 \mathrm{~kg} / \mathrm{d}$ (Rajala-Schultz et al., 1999). For ketosis, for cows in parity 4 and higher, an average loss of $353.4 \mathrm{~kg}$ of milk per cow per 305-d lactation yield was estimated by Rajala-Schultz et al. (1999). For elevated SCC due to clinical or subclinical mastitis, the loss in milk yield was between 2 to $6 \mathrm{~kg}$ of milk per day (Windig et al., 2005). Drops in yield were more frequent in cows compared with heifers. In the first lactation, $56.9 \%$ of cows had a drop in daily milk yield of $2 \mathrm{~kg}, 30 \%$ had a drop of $4 \mathrm{~kg}$, and $9 \%$ had a drop of $6 \mathrm{~kg}$ associated with high SCC. In higher-parity cows, these percentages were $68.5,40.8$, and $15.1 \%$, respectively (Windig et al., 2005). The recovery also differs between the diseases, in time as well as in yield level that is reached after recovery (Rajala-Schultz et al., 1999; Windig et al., 2005; Onyiro et al., 2008).

In an earlier study, weak genetic associations were found between the variance in test-day milk yields and 
udder health and longevity (H. A. Mulder, unpublished data). The weak associations were likely due to the fact that test-day intervals are 4 to $6 \mathrm{wk}$. As a consequence, the effect of health issues on test-day milk yield could be small, especially when the health issue is short, subclinical, and in the middle of a test-day interval. Presently, many farmers record milk yields of every milking, using automatic milking systems (AMS) or electronic milk measurement systems. Fluctuations in daily milk yield of individual cows can be determined and the association between fluctuations in milk yield and animal diseases can be estimated more precisely compared with test-day records. Because animal diseases often cause drops in milk yield, fluctuations in daily milk yield are expected to be useful indicator traits for genetic evaluation of health and longevity in dairy cattle. So far, no research has been done on how to use fluctuations in daily milk yield as indicator traits for genetic evaluation of cow health. Therefore, the aim of our study was to estimate the heritability of various simple traits related to fluctuations in milk yield, to estimate genetic correlations among these fluctuation traits, and to estimate genetic correlations between these fluctuations traits with health and functional traits for which routine breeding values are estimated. Furthermore, we calculated partial genetic correlations and relative contributions to reliability to investigate which of the new traits is best at predicting fertility, health, and longevity. This study is the first exploring how daily milk yield records of AMS can be used to derive traits to improve fertility, health, and longevity of cows, and we discuss which steps are needed to implement such a trait for routine genetic evaluation.

\section{MATERIALS AND METHODS}

\section{Data}

The original data set for this study was obtained from CRV (Arnhem, the Netherlands) and contained 331,520 lactations collected from 1997 to 2016. The data consisted of records from AMS. Records of in total 182,064,264 milkings were provided with cow identification, milk yield in kilograms, the date, and the start and end time of each milking. Records for parity 1 were extracted. Cows that were at least $75 \%$ Holstein were used in the analysis. Cows were required to have records for at least 21 consecutive days and all records were used until d 335 after calving. To adjust for herd, year of calving, and season of calving, herdyear-season (HYS) classes were made with 20 possible years of calving (1997-2016) and 2 possible seasons of calving (January-June and July-December). The use of 3 or 4 seasons in the HYS decreased the data set by 15 and $20 \%$, respectively, when the requirement of $\geq 5$ lactations per HYS was used. With 2 seasons per year, the loss of data was only $2 \%$. After the data edits, 498 herds with 67,025 lactations were used for analysis. Besides the milk yield data, the complete pedigree of the cows was available as well as the birthdates and the calving dates per parity for every cow.

Daily milk yield records were calculated based on the milk yields and the start times of each AMS milking (on average 2.8 times a day). Milking intervals in minutes between 2 consecutive milkings were calculated from the start time of the milking to the start time of the next milking. Milk yield of each single milking was converted to a 24-h day yield, based on kilograms of milk produced per minute using the time interval between 2 consecutive milkings. Subsequently, a day milk yield was calculated as the weighted average milk yield using the milk yields of each milking on that day and the length of the time intervals between consecutive milkings as weight factors. For the intervals longer than $1 \mathrm{~d}$ (>1,440 min), the milking was considered as missing value and not considered when calculating the weighted average day yield. When the day yield was higher than the average day yield of the whole data set plus 4 times the standard deviation of the whole data set, the day yield was considered as an outlier and therefore also set to a missing value.

\section{Defining Fluctuations in Milk Yield}

Fluctuations in milk yield were defined in 3 ways: the number of drops in milk yield during lactation either based on (1) a rolling average or (2) a regression and (3) the natural logarithm of the variance of milk yield of an individual cow per lactation.

For the rolling average method, the day yield was tested against the average of the preceding $7 \mathrm{~d}$ before the day of interest (d 8). Consequently, the first test was on $\mathrm{d} 8$ in lactation, and the first $7 \mathrm{~d}$ were used to set the average for testing the next day yield. The Student's $t$-test for the rolling average was $t=(y-\bar{\mu}) /(\mathrm{SD} / \sqrt{n})$, where $y$ is the $\mathrm{d} 8$ yield, $\bar{\mu}$ is the rolling average over $7 \mathrm{~d}$, SD is the standard deviation of the 7-d yields, and $n$ is the number of day yields in the rolling average $(n=7)$. A significance threshold $(\alpha)$ of $1 \%$ was used for testing for a significant decreased day yield. The threshold $1 \%$ was arbitrarily chosen. The rolling average based on $7 \mathrm{~d}$ had 6 degrees of freedom (n - 1). A drop in day yield was significant when $t$ $\leq-3.143$ (Ott and Longnecker, 2010). In preliminary research, we investigated also thresholds of 5 and $0.1 \%$. Using a $0.1 \%$ threshold resulted in too few drops, and therefore heritabilities were very low, whereas results in 
terms of correlations were similar when using either a threshold of 1 or $5 \%$ threshold.

For the regression method, a 1-sided Student's $t$-test was performed per day on the regression coefficient over the last $7 \mathrm{~d}$ yields. In a 7-d interval, it can be expected that the regression coefficient is generally near zero in absence of diseases or other disturbances, except for the start of lactation. Therefore, the regression coefficient was tested for a significant and negative deviation from zero. The Student's $t$-test used to test whether the regression coefficient was significantly below zero was $t=$ $(\beta 1-0) / \mathrm{SE}(\beta 1)$, where $\beta 1$ is the regression coefficient over 7 -d yields and $\mathrm{SE}(\beta 1)$ is the standard error of this regression coefficient (Ott and Longnecker, 2010). Again, a significance threshold of $1 \%(\mathrm{t} \leq-5.59 ; 5 \mathrm{df})$ was used to test for a significant drop in yield (Ott and Longnecker, 2010). The period of $7 \mathrm{~d}$ to estimate the rolling average or the regression coefficient was chosen as a balance between the accuracy of estimating the rolling average or regression coefficient and having enough opportunity to detect sudden changes in milk production. Subsequently, each day got a binary score; days with a significant drop in milk yield were scored with a 1 and the other days were scored as zero. Therefore, the number of drops per lactation was simply the sum of the binary scores (see Figure 1).

Three new traits were defined. First, drop rolling average, the sum of the binary scores for the rolling average method; second, drop regression, the sum of the binary scores for the regression method; and third, the log-transformed variance of day yield (LnVar), which was calculated based on the whole lactation up to $\mathrm{d}$ 335 and was based on at least $21 \mathrm{~d}$. The variance was log-transformed to make it more normally distributed. By using the natural logarithm, the underlying model for the variance was an exponential model, which made estimates of genetic variance directly comparable to results from other studies using an exponential model for the residual variance (Sell-Kubiak et al., 2015).

\section{Genetic Analysis}

For the 3 new defined traits, heritabilities and breeding values were estimated as well as the phenotypic and genetic correlations between the traits using ASReml4.1 (Gilmour et al., 2014). Estimation of heritabilities and breeding values was based on univariate analyses with the linear animal model

$$
\mathbf{y}=\mathbf{X} \boldsymbol{\beta}+\mathbf{Z a}+\mathbf{e},
$$

where $\mathbf{y}$ is a vector of observations; $\mathbf{X}$ is the incidence matrix of the fixed effects; $\boldsymbol{\beta}$ is a vector of the fixed effects, which were HYS, year-month of calving, DIM class first day in lactation, DIM class last day in lactation, lactation length, age of calving, and age of calving squared; $\mathbf{Z}$ is the incidence matrix relating observations to additive genetic effects or breeding values; $\mathbf{a}$ is the

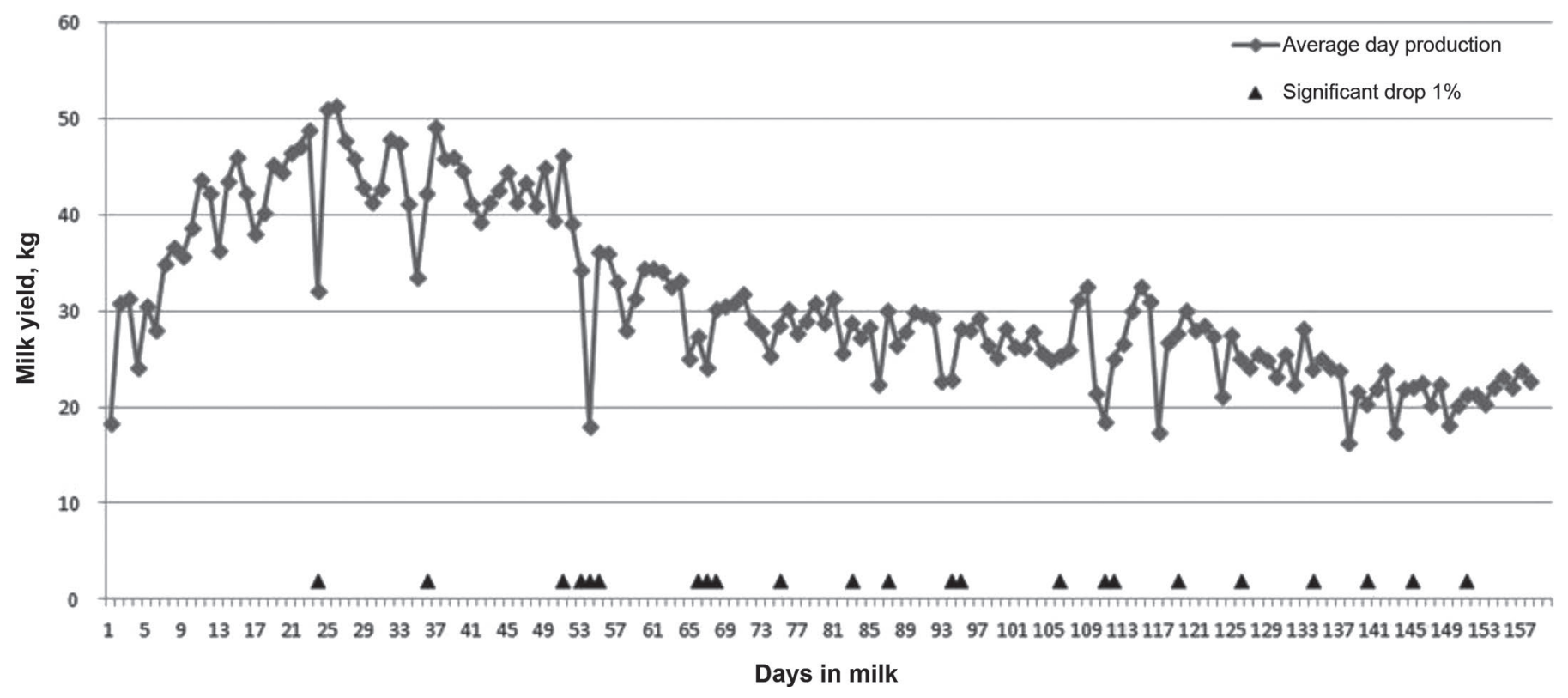

Figure 1. A visualization of a lactation curve from the data with an indication of the significant dropped day yields. The drops in this figure are based on the rolling average method. 
vector of the random animal additive genetic effects or breeding values $\mathbf{a} \sim N\left(\mathbf{0}, \mathbf{A} \sigma_{a}^{2}\right)$, where $\mathbf{A}$ is the numerator relationship matrix and $\sigma_{a}^{2}$ is the additive genetic variance; and $\mathbf{e}$ is a vector of random residuals $\mathbf{e} \sim N\left(\mathbf{0}, \mathbf{I} \sigma_{e}^{2}\right)$, where $\sigma_{e}^{2}$ is the residual variance and $\mathbf{I}$ is the identity matrix. The A matrix contained 257,512 animals. The interaction year-month of calving was added to the model to correct for the month of calving, as the HYS classes were only based on 2 seasons. To correct for lactations that were not complete in the data set, the classes of DIM of the first day and the last day of the lactation were added to the model. In total, 2,662 cows started in the AMS after d 35, mainly due to introduction of AMS in the farm, whereas 17,872 cows ended their lactation before d 275, likely due to culling. The maximum lactation length was $335 \mathrm{~d}$ in lactation, in total there were 11 classes. The first class considered d 0 to 35, the following classes contained all $30 \mathrm{~d}$, so the second class contained d 36 to 65 . The division was the same for DIM effects of the last day.

Genetic correlations among the new defined fluctuation traits and average daily milk yield were estimated with bivariate animal models

$$
\left[\begin{array}{l}
\mathbf{y}_{1} \\
\mathbf{y}_{2}
\end{array}\right]=\left[\begin{array}{cc}
\mathbf{X}_{1} & \mathbf{0} \\
\mathbf{0} & \mathbf{X}_{2}
\end{array}\right]\left[\begin{array}{l}
\mathbf{b}_{1} \\
\mathbf{b}_{2}
\end{array}\right]+\left[\begin{array}{cc}
\mathbf{Z}_{1} & \mathbf{0} \\
\mathbf{0} & \mathbf{Z}_{2}
\end{array}\right]\left[\begin{array}{l}
\mathbf{a}_{1} \\
\mathbf{a}_{2}
\end{array}\right]+\left[\begin{array}{l}
\mathbf{e}_{1} \\
\mathbf{e}_{2}
\end{array}\right],
$$

where $\mathbf{y}_{i}$ are the observations for the traits 1 and 2, where $i$ stands for trait 1 or trait $2 ; \mathbf{X}_{i}$ is the incidence matrix for the fixed effects of trait $i$; $\mathbf{b}_{i}$ is the vector with fixed effects for trait $i$; $\mathbf{Z}_{i}$ is the incidence matrix for the random effects for trait $i$; $\mathbf{a}_{i}$ the vector with random additive genetic effects or breeding values for trait $i$; and $\mathbf{e}_{i}$ the vector with the random residual effects for trait $i$. The breeding values and residuals were assumed bivariate normally distributed

$$
\begin{gathered}
{\left[\begin{array}{l}
\mathbf{a}_{1} \\
\mathbf{a}_{2}
\end{array}\right] \sim N\left[\left(\begin{array}{l}
\mathbf{0} \\
\mathbf{0}
\end{array}\right), \mathbf{A} \otimes\left(\begin{array}{cc}
\sigma_{a_{1}}^{2} & \sigma_{a_{1} a_{2}} \\
\sigma_{a_{1} a_{2}} & \sigma_{a_{2}}^{2}
\end{array}\right)\right] \text { and }} \\
{\left[\begin{array}{l}
\mathbf{e}_{1} \\
\mathbf{e}_{2}
\end{array}\right] \sim N\left[\left(\begin{array}{l}
\mathbf{0} \\
\mathbf{0}
\end{array}\right), \mathbf{I} \otimes\left(\begin{array}{cc}
\sigma_{e_{1}}^{2} & \sigma_{e_{1} e_{2}} \\
\sigma_{e_{1} e_{2}} & \sigma_{e_{2}}^{2}
\end{array}\right)\right],}
\end{gathered}
$$

where $\sigma_{a_{i}}^{2}$ is the additive genetic variance for trait $i$; $\sigma_{a_{1} a_{2}}$ is the additive genetic covariance between trait 1 and $2 ; \sigma_{e_{i}}^{2}$ is the residual variance for trait $i$; and $\sigma_{e_{1} e_{2}}$ is the residual covariance between trait 1 and 2 .

\section{Genetic Correlations with Health, Longevity, and Persistency Traits}

Approximate genetic correlations between the new fluctuation traits $(f)$ and existing fertility, health, and longevity traits $(h)$ were calculated using the Calo method (Calo et al., 1973; Blanchard et al., 1983), correcting the Pearson correlation between EBV of bulls with their respective reliabilities

$$
\rho_{f, h}=\frac{\sqrt{\left(\sum R i h_{f}^{2}\right)\left(\sum R i h_{h}^{2}\right)}}{\sum R i h_{f}^{2} R i h_{h}^{2}} \times r_{f, h},
$$

where $R i h_{i}^{2}$ is the reliability for trait $i$ and $r_{f, h}$ is the Pearson correlation between EBV of the 2 traits. The fertility, health, and longevity traits used for the analysis were calving interval (CaI), interval first last insemination (FL; CRV, 2015), direct and maternal survival at birth (CRV, 2011), udder health (UDH; CRV, 2016), claw health (CLW; CRV, 2014), ketosis (Vosman et al., 2015), and longevity (CRV, 2012b). The $\mathrm{EBV}$ for CaI and FL were the overall EBV for CaI and FL weighing the EBV for CaI and FL in parities 1, 2, and 3; each of these EBV were estimated with a multiple-trait breeding value estimation consisting of 34 traits (CRV, 2015). The EBV for direct and maternal survival were based on calf records in first-parity cows (CRV, 2011). The EBV for UDH was based on the EBV for clinical and subclinical mastitis; both EBV are the overall EBV weighing the EBV in the 3 parities. The traits used in the breeding value estimation were SCS derived traits and records of clinical mastitis (CRV, 2016). The EBV for CLW was based on hoof trimming records for sole hemorrhage, digital dermatitis, interdigital dermatitis, sole ulcer, interdigital hyperplasia, and white line disease; the EBV of parity 1 and older cows were combined into 1 index (CRV, 2014). The EBV for ketosis was the overall EBV for ketosis weighing the EBV for ketosis in the 3 parities. The EBV were based on the ketosis indicator, which is based on Fourier transform infrared measurements for milk acetone, milk BHB levels, and the milk fat-toprotein ratio (Vosman et al., 2015). The EBV for longevity was based on survival analysis of life span records (CRV, 2012b). Furthermore, the EBV for persistency (PER; CRV, 2012a) was used in this analysis because it is expected that persistent cows show less fluctuations in milk yield. The EBV for PER was defined as the difference in sum of the EBV for fat and protein yield from d 61 to 305, with the EBV for fat and protein yield at d 60 times $245 \mathrm{~d}$. The EBV per day are obtained from a random regression test-day model. The 
EBV for PER was basically the decline in fat and protein yield after d 60 (CRV, 2012a). In this analysis, EBV of 222 bulls were used that had reliabilities of at least 0.5 for any of the traits and at least 5 daughters in the data set.

To investigate which of the 3 fluctuation traits added the most new information to predict fertility, health, and longevity independent of persistency or average milk yield, we performed 2 additional analyses: partial genetic correlations controlling persistency or average milk yield and the relative contribution of the fluctuation trait to predict health or longevity (fertility was excluded because of lower correlations) when using an index with the true breeding values of average milk yield, persistency, and the fluctuation trait. Furthermore, LnVar was the raw within-cow variance and, therefore, it was expected that high-yielding cows or less-persistent cows would have a higher variance due to a higher difference between peak yield and yield at the start and end of lactation. These 2 analyses corrected LnVar for the effects of average milk yield and persistency in its relationship to fertility, health, and longevity.

First, partial genetic correlations were calculated as

$$
r_{x y, z}=\frac{r_{x y}-r_{x z} r_{y z}}{\sqrt{1-r_{x z}^{2}} \sqrt{1-r_{y z}^{2}}},
$$

where case trait $x$ is the fluctuation trait; $y$ is the fertility, health, or longevity trait; and $z$ is average milk yield or persistency. All used genetic correlations were either based on genetic correlations obtained from the bivariate analyses or based on approximate genetic correlations using the Calo method.

Second, the relative contributions of the fluctuations traits to predict breeding values for health or longevity were calculated for a selection index including average milk yield, persistency, and 1 fluctuation trait. The analyses were based on using the true breeding values in an index; the additive genetic variances of all traits were set to 1 . Selection index weights were calculated as $\mathbf{b}=\mathbf{P}^{-1} \mathbf{g}$, where $\mathbf{P}$ is in this case the correlation matrix of the breeding values for average milk yield, persistency, and 1 fluctuation trait, and $\mathbf{g}$ is the vector of correlations between average milk yield, persistency, and 1 fluctuation trait with a particular health or longevity trait. The proportion of explained variance or reliability was calculated as $\mathrm{R}^{2}=\mathbf{b}^{\prime} \mathbf{P b}=\mathbf{b}^{\prime} \mathbf{g}$ and the proportion of variance explained by each of the 3 traits in the index was calculated as

$$
\mathrm{R}^{2} \operatorname{prop}_{x}=\operatorname{abs}\left(b_{x} g_{x}\right) / \sum_{i=1}^{3} \operatorname{abs}\left(b_{i} g_{i}\right)
$$

where $\mathrm{R}^{2} \operatorname{prop}_{x}$ is the proportion of $\mathrm{R}^{2}$ explained by trait $x$. The absolute values of $b_{x} g_{x}$ were used because, in some cases, the product was negative. Furthermore, in the denominator $\sum_{i=1}^{3} \operatorname{abs}\left(b_{i} g_{i}\right)$ was used, so that the sum of the $3 \mathrm{R}^{2} \operatorname{prop}_{x}$ was 1 . The analyses were performed to predict udder health, ketosis, and longevity because they had the highest absolute genetic correlations with the 3 fluctuation traits.

\section{RESULTS}

\section{Descriptive Statistics}

Table 1 presents summary statistics of the fluctuation traits and average milk yield. Figure 2 shows the distributions of the fluctuation traits. The means of drop traits differed between the rolling average method and the regression method, as well as the maximum number of significant drops found per lactation. The rolling average method identified more drops than the regression method. Both drop traits showed a bimodal distribution, with a small peak around zero drops and a peak close to the average number of drops. The LnVar was approximately normally distributed.

\section{Heritabilities and Genetic Correlations Among Fluctuation Traits}

The genetic parameters for the 3 fluctuation traits and average milk yield are presented in Table 2. The heritabilities for drop traits were $0.08(\mathrm{SE}=0.01)$ and $0.06(\mathrm{SE}=0.01)$ for the rolling average and the regression method, respectively. For LnVar, the heritability was $0.10(\mathrm{SE}=0.01)$. The difference in variance components between the drop traits and LnVar can be

Table 1. Mean, SD, minimum, and maximum for the fluctuation traits milk yield drop rolling average and drop regression as well as log-transformed variance (LnVar) and average milk yield (AMY)

\begin{tabular}{lcccc}
\hline Trait & Mean & SD & Minimum & Maximum \\
\hline Drop rolling average & 46.89 & 19.66 & 0 & 109 \\
Drop regression & 12.51 & 8.26 & 0 & 64 \\
LnVar & 3.11 & 0.77 & -4.21 & 7.66 \\
AMY, kg/d & 27 & 5.67 & 0.2887 & 74.62 \\
\hline
\end{tabular}


Table 2. Estimated $\mathrm{h}^{2}$, additive genetic $\left(\sigma_{a}^{2}\right)$ and residual variances $\left(\sigma_{e}^{2}\right)$, with $\mathrm{SE}$ for the fluctuations traits milk yield drop rolling average and drop regression as well as log-transformed variance (LnVar) and average milk yield (AMY)

\begin{tabular}{llll}
\hline Item & \multicolumn{1}{c}{$\mathrm{h}^{2}(\mathrm{SE})$} & \multicolumn{1}{c}{$\sigma_{a}^{2}(\mathrm{SE})$} & $\sigma_{e}^{2}(\mathrm{SE})$ \\
\hline Drop rolling average & $0.08(0.008)$ & $3.44(0.37)$ & $41.96(0.36)$ \\
Drop regression & $0.06(0.007)$ & $2.21(0.27)$ & $34.74(0.28)$ \\
LnVar & $0.10(0.010)$ & $0.03\left(3.13 \times 10^{-3}\right)$ & $0.27\left(2.76 \times 10^{-3}\right)$ \\
AMY & $0.39(0.014)$ & $7.62(0.32)$ & $12.15(0.22)$ \\
\hline
\end{tabular}

explained by the different scales and ranges of observations. For average milk yield, heritability was 0.39 (SE $=0.01$ ).

The genetic correlations among the 3 new traits and average milk yield are presented in Table 3 . The genetic correlation between the 2 drop traits was 0.87 ( $\mathrm{SE}=$ $0.03)$, showing that these 2 traits are genetically very similar. The genetic correlation of LnVar with the drop traits was much lower. The genetic correlation between drop rolling average with LnVar was $0.25(\mathrm{SE}=0.07)$ and between drop regression with LnVar was 0.23 (SE $=0.07$ ). This shows that LnVar is genetically a different trait compared with the drop traits. Furthermore, the genetic correlations between the drop traits and average milk yield were negative, indicating that cows with higher milk yield have genetically fewer drops in milk yield. On the contrary, the genetic correlation between LnVar and average milk yield was 0.61 (SE $=0.04$ ), showing that cows with higher milk production have a higher variance in milk yield. In summary, LnVar was genetically a different trait than the drop traits, whereas the drop traits were genetically similar.

\section{Approximate Genetic Correlations with Health, Longevity, and Persistency Traits}

Approximate genetic correlations between the fluctuation traits and fertility, health, and longevity traits were calculated to investigate the extent to which fluctuation traits were associated with genetic differences in fertility, health, and longevity (Table 4). A negative correlation between the fluctuation traits and the health traits was expected, as the EBV for the fluctuation traits were higher when more fluctuations appeared in a lactation, which was assumed to be associated with poor health (i.e., a lower EBV for health traits). Indeed, most genetic correlations were negative. The strongest genetic correlation found was between LnVar and ketosis $(-0.52)$, whereas lower but still favorable genetic correlations were found between LnVar and CaI $(-0.20)$, FL $(-0.12)$, UDH $(-0.36)$, CLW $(-0.07)$, and longevity (-0.30). In most cases, LnVar had the strongest (negative) correlations compared with the 2 drop traits. Strikingly, positive genetic correlations between the drop traits (both methods) and the fertility traits CaI and FL were found, whereas LnVar had a negative genetic correlation with $\mathrm{CaI}$ and $\mathrm{FL}$, again indicating that LnVar was genetically different than the drop traits. Persistency showed for all 3 fluctuation traits a moderate negative genetic correlation indicating that persistent cows have fewer fluctuations in milk yield.

\section{Finding the Best Predictor}

To find the best predictor trait, partial genetic correlations and relative contributions to reliability of fertility, health, or longevity were calculated. Partial genetic correlations between the fluctuations traits and fertility, health, and longevity when controlling for persistency or average milk yield are shown in Table 5, whereas relative contributions to reliability are shown in Table 6. Partial genetic correlations were in most cases only slightly different than the original approximate genetic correlations (Table 4). The largest changes were observed for the partial correlations between the drop traits and longevity. Even when controlling for persistency or average milk yield, LnVar had the strongest correlations with udder health, ketosis, and longevity

Table 3. The genetic (above diagonal) and residual (below diagonal) correlations between the 3 fluctuation traits milk yield drop rolling average and drop regression, log-transformed variance (LnVar), and average milk production (AMY; SE between parentheses)

\begin{tabular}{lcccr}
\hline Item & Drop rolling average & Drop regression & LnVar & \multicolumn{1}{c}{ AMY } \\
\hline Drop rolling average & $0.58(0.004)$ & $0.87(0.03)$ & $0.25(0.07)$ & $-0.35(0.05)$ \\
Drop regression & $0.21(0.006)$ & $0.21(0.006)$ & $0.23(0.07)$ & $-0.08(0.06)$ \\
LnVar & $0.06(0.009)$ & $0.06(0.009)$ & $0.20(0.009)$ & $0.61(0.04)$ \\
AMY & & & \\
\hline
\end{tabular}


Drop rolling average

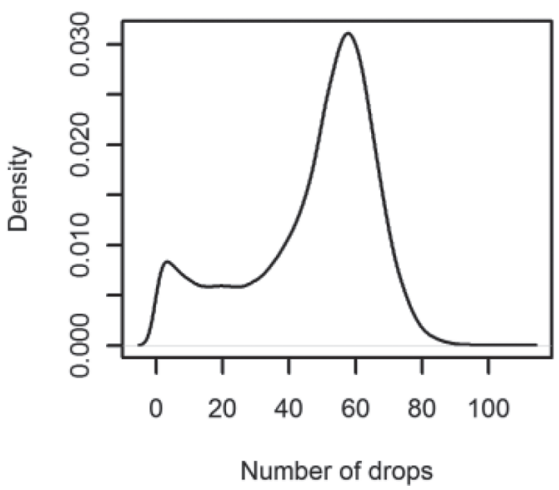

Drop regression

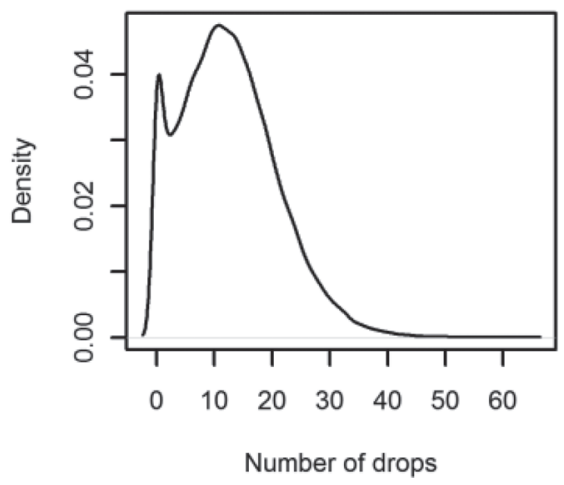

distribution LNvar

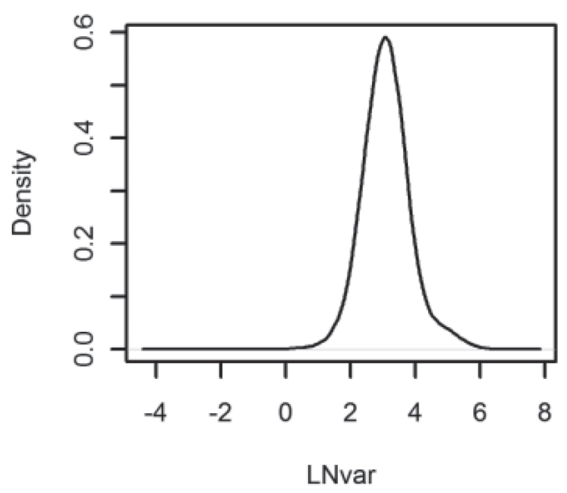

Figure 2. Distributions of milk yield drop rolling average and drop regression as well as log-transformed variance (LnVar).

compared with the drop traits. When predicting udder health, ketosis, or longevity (Table 6) with an index of average milk yield, persistency, and 1 fluctuation trait, the reliability of the index with LnVar was substantially higher when predicting udder health or ketosis than when using the drop traits; however, in all cases reliabilities were low. For longevity, the reliability of the index with drop regression was slightly better than with LnVar. The relative contributions to reliability of LnVar were for all 3 traits above $75 \%$, indicating that LnVar contains new information in comparison to persistency and average milk yield when predicting udder health, ketosis, and longevity. These results show that LnVar was the best trait among the fluctuation traits to predict udder health, ketosis, and longevity.

\section{DISCUSSION}

We investigated 3 traits related to fluctuations in milk yield and found that they have heritabilities in the range of 0.06 to 0.10 . Among the 3 fluctuation traits defined in our study, we found that LnVar was the best trait to predict udder health, ketosis, and longevity.

\section{Definition and Heritability of Fluctuations}

The heritability for drop based on the regression method was lower than with the rolling average method, likely due to the lower number of detected significant drops in milk production. The regression method seemed less sensitive to detect drops in milk production. One reason is simply that having a single day with a lower milk yield may not be enough to give rise to a significant drop because the regression coefficient is based on $7 \mathrm{~d}$. The rolling average method, on the other hand, tests directly the individual day milk yield with the previous $7 \mathrm{~d}$. A lower sensitivity to detect drops may result in less false positives, but may also result in more false negatives. The same is true when decreasing the significance threshold from 0.05 to 0.01 or 0.001 , that less false positives are detected (type I error), whereas the number of false negatives also increases (type II error). Type I and type II errors are inversely correlated, when one of them decreases the other will increase (Ott and Longnecker, 2010). We observed that a more stringent threshold decreased the heritability (results not shown) and, therefore, standard

Table 4. Approximate genetic correlations between the 3 fluctuation traits milk yield drop rolling average and drop regression, log-transformed variance (LnVar), and average milk production (AMY) with fertility, health, persistency, and longevity traits ${ }^{1,2}$ using correlations between EBV adjusted for reliabilities

\begin{tabular}{lcccccrrrr}
\hline Item & CaI & FL & DS & MS & UDH & CLW & KET & LON & PER \\
\hline Drop rolling average & $0.20^{3}$ & 0.22 & -0.06 & 0.13 & -0.09 & -0.03 & -0.20 & -0.08 & -0.62 \\
Drop regression & 0.27 & 0.27 & -0.02 & 0.20 & -0.10 & 0.15 & -0.15 & 0.10 & -0.39 \\
LnVar & -0.20 & -0.12 & -0.05 & 0.02 & -0.36 & -0.07 & -0.52 & -0.30 & -0.29 \\
AMY & -0.20 & -0.18 & -0.09 & 0.05 & -0.12 & -0.06 & -0.25 & -0.02 & 0.36 \\
\hline
\end{tabular}

${ }^{1} \mathrm{CaI}=$ calving interval; $\mathrm{FL}=$ interval from first to last insemination; $\mathrm{DS}=$ direct survival at birth; $\mathrm{MS}=$ maternal survival at birth; UDH $=$ udder health index; CLW = claw health index; KET = ketosis; LON = longevity; PER = persistency.

${ }^{2}$ The EBV for fertility, health, persistency, and longevity traits are presented as higher being better, whereas higher EBV for the fluctuation traits are worse. Therefore, negative genetic correlations are favorable.

${ }^{3}$ Approximate standard errors of Pearson correlations are between 0.04 and 0.07 . 
Table 5. Partial genetic correlations between the 3 fluctuation traits and fertility, health, and longevity ${ }^{1,2}$ controlling for persistency or average milk yield

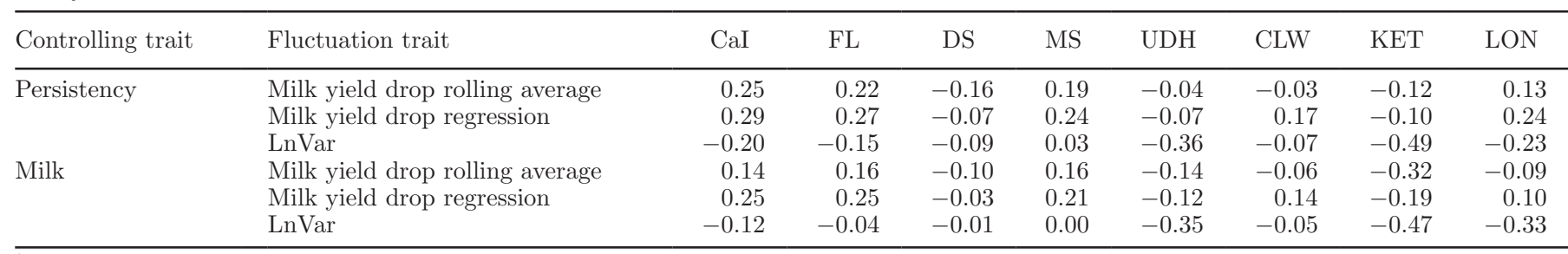

${ }^{1} \mathrm{CaI}=$ calving interval; $\mathrm{FL}=$ interval from first to last insemination; $\mathrm{DS}=$ direct survival at birth; $\mathrm{MS}=$ maternal survival at birth; UDH $=$ udder health index; CLW = claw health index; KET = ketosis; LON = longevity.

${ }^{2}$ The EBV for fertility, health, persistency, and longevity traits are presented as higher being better, whereas higher EBV for the fluctuation traits are worse. Therefore, negative genetic correlations are favorable.

errors on estimated genetic parameters also increased and estimated genetic correlations were more variable and less consistent. The reason for the decline in heritability with more stringent thresholds is likely that more animals have zero drops in milk production when the threshold is more stringent. The drop traits behave similarly to binary traits (i.e., infected; not infected), with a lower heritability on the observed scale when the incidence is lower (Falconer and Mackay, 1996). A threshold of 0.01 was considered best given the combination of heritabilities and a consistent pattern of genetic correlations with diseases.

In the current study, the drop traits were based on the number of days with a significantly reduced milk yield. Often drops in milk production appear as episodes of a few days or longer with reduced milk yield. In preliminary research, we also investigated the number of episodes with at least $2 \mathrm{~d}$, episodes of 1,2 , or $3 \mathrm{~d}$, or episodes that are $4 \mathrm{~d}$ or longer. Most traits were phenotypically highly correlated, with correlations above 0.9 ; even in cases with lower phenotypic correlations the genetic correlations appeared to be very high (i.e., higher than 0.85), indicating that these other trait definitions were genetically almost identical. Therefore, we decided to restrict further research on the 3 fluctuation traits shown here.

The drop traits had unexpectedly favorable genetic correlations to average milk yield, indicating that cows with high milk production had fewer drops in milk yield. Phenotypically, positive correlations would be expected because higher health would result in fewer drops in milk production and, therefore, a higher average milk yield. However, genetically, higher average milk yield is associated with lower health, as also observed in our study (Table 4); therefore, it was expected that high-yielding cows would have had more drops in milk production due to their higher genetic predisposition to get diseases such as mastitis and ketosis. A possible reason is that, due to the strong positive genetic correlation between average milk yield and the variance of milk yield (LnVar), some drops in milk production were not significant because of high variance resulting in a lower test statistic of the Student's $t$-test. This may partly explain why the drop traits were less useful as predictors of health and longevity.

Another unexpected result was that drop traits positively correlated to $\mathrm{CaI}$ and FL, whereas LnVar was negatively correlated to $\mathrm{CaI}$ and FL. It seemed that

Table 6. The relative contributions of the 3 fluctuation traits [milk yield drop rolling average and drop regression as well as log-transformed variance (LnVar)] to reliability and the overall reliability $\left(\mathrm{R}^{2}\right)$ to predict udder health (UDH), ketosis (KET), or longevity (LON) using an index with the true breeding values for average milk yield (AMY), persistency, and a fluctuation trait

\begin{tabular}{llcccc}
\hline & & \multicolumn{2}{c}{ Relative contribution to reliability ${ }^{1}\left(\mathrm{R}^{2}\right)$} & \\
\cline { 3 - 4 } Trait to predict & Fluctuation trait & Milk & Persistency & Fluctuation trait & \multirow{2}{*}{$\mathrm{R}^{2}$} \\
\hline UDH & Drop rolling average & 0.57 & 0.21 & 0.22 & 0.04 \\
UDH & Drop regression & 0.54 & 0.28 & 0.18 & 0.04 \\
UDH & LnVar & 0.15 & 0.07 & 0.79 & 0.17 \\
KET & Drop rolling average & 0.57 & 0.17 & 0.27 & 0.17 \\
KET & Drop regression & 0.61 & 0.31 & 0.08 & 0.14 \\
KET & LnVar & 0.11 & 0.04 & 0.85 & 0.28 \\
LON & Drop rolling average & 0.02 & 0.90 & 0.08 & 0.10 \\
LON & Drop regression & 0.02 & 0.81 & 0.17 & 0.15 \\
LON & LnVar & 0.02 & 0.22 & 0.76 & 0.14 \\
\hline
\end{tabular}

${ }^{1}$ See equation 1 . 
cows with better fertility would have more drops, possibly because they show heat better and therefore have reduced milk production for a day or longer. This may be another reason why the drop traits were less useful as predictors for health and longevity than LnVar.

The third fluctuation trait used in this study was LnVar, the within-cow log-transformed variance of daily milk yield. The advantage of this measure is that no significance testing is involved. Furthermore, the estimate of the genetic variance on the natural log scale is comparable to many studies estimating genetic variance in environmental variance using an exponential model on the residual variance (Hill and Mulder, 2010). The estimated genetic variance of 0.03 agrees very well with earlier studies on milk yield (Mulder et al., 2013; Rönnegård et al., 2013; Vandenplas et al., 2013). The heritability of LnVar is higher than the heritability of environmental variance for many traits because our measure is based on $\sim 300$ records per cow. In most studies on genetics of environmental or residual variance the heritability is defined on the individual record level, yielding heritabilities of 0.01 or lower in dairy cattle (Mulder et al., 2013; Rönnegård et al., 2013; Vandenplas et al., 2013). The heritability for LnVar is comparable to the heritability of the within-individual variance for egg color in purebred laying hens, which was $\sim 0.15$ (Mulder et al., 2016), and the heritability of the within-litter variance of piglet birth weight, which varied between 0.08 and 0.14 in different studies (Damgaard et al., 2003; Kapell et al., 2011; Sell-Kubiak et al., 2015). Furthermore, the high genetic correlation between LnVar and average milk yield of 0.61 is in agreement with Mulder et al. (2013), who found a genetic correlation of 0.63 between milk yield and residual variance in Swedish Holsteins using a double hierarchical generalized linear model for modeling differences in residual variance.

Although LnVar is easy to use, a major drawback is that LnVar is a composite measure of residual variance and the shape of the lactation curve. For instance, cows with a high peak yield and low persistence will have a higher variance than cows with a low peak yield and high persistency. To get a more true measure of residual variance, modeling an individual cow's lactation curve would be a logical next step in research and toward routine genetic evaluation, but caution is needed to avoid the lactation curve itself taking away information with respect to occurrence of diseases or other disturbances. Smoothing methods can be used, such as in Codrea et al. (2011), to get a measure of the residual variance rather than the raw variance as used in the current study. Alternative methods are random regression test-day models combined with double hierarchical generalized linear models (Vandenplas et al.,
2013); however, results of partial correlations controlling for persistency and average milk yield showed that the relationships between LnVar and fertility, health, and longevity were not much affected by persistency or average milk yield. Furthermore, calculations of relative contributions to predict udder health, ketosis, and longevity clearly showed that LnVar contains additional information about health and longevity beyond average milk yield and persistency. Therefore, refining LnVar by accounting for lactation curve may yield only small improvements in reliability of health and longevity in parity 1, but may yield larger improvements in parity 2 and further due to much higher peak yields in older cows.

For all 3 traits, the heritabilities were similar to heritabilities found for animal diseases or its indicators. Frequent diseases, such as mastitis, have heritabilities in the range of 0.01 to 0.05 , whereas the indicator trait SCS has heritabilities in the range of 0.1 to 0.2 (Heringstad et al., 2000; Rupp and Boichard, 2003). Ketosis has also a high incidence, especially occurring in later parities, and has a low heritability in the range of 0.01 to 0.08 (Kadarmideen et al., 2000; Koeck et al., 2013), whereas the indicator for ketosis in milk, BHB, has heritabilities in the range of 0.1 to 0.3 (van der Drift et al., 2012; Koeck et al., 2014). Claw disorders have heritabilities in the range of 0.01 to 0.14 (van der Waaij et al., 2005; van der Linde et al., 2010; Häggman and Juga, 2013). Metritis and displaced abomasum are less frequent, resulting in low heritabilities of 0.01 to 0.05 (Koeck et al., 2012; Parker-Gaddis et al., 2014). Indicator traits have often higher heritabilities than the direct disease traits, mainly because the indicator trait is continuous and not binary. The fluctuations traits in our study may, therefore, increase the reliability of existing EBV for health and longevity; however, due to moderate to low genetic correlations, improvements are expected to be small, because for the most frequent diseases, such as mastitis and ketosis, other good indicator traits are already available. The fluctuation traits we studied reflect multiple diseases, as also indicated by the estimated genetic correlations, and may relate more to subclinical diseases or to general disease resistance or resilience.

\section{Variance as Resilience Indicator}

As stated, the value of using the variance of milk yield in breeding is expected to be more related to improving general disease resistance or resilience. Disease resilience is defined as the ability to maintain performance during infectious challenges (Doeschl-Wilson et al., 2012). Simulations by Mulder et al. (2015) showed that EBV for residual variance can capture additive 
Table 7. The average EBV for health, persistency, and longevity traits for the best 20 and the worst 20 sires selected on their EBV for logtransformed variance $(\text { LnVar) })^{1,2}$

\begin{tabular}{lccccccccc}
\hline Item & CaI & FL & DS & MS & UDH & CLW & KET & PER & LON \\
\hline Best sires & 102 & 100 & 99 & 101 & 104 & 101 & 105 & 105 & 298 \\
Worst sires & 100 & 100 & 99 & 100 & 99 & 101 & 98 & 102 & 92 \\
$P$-value Student $t$-test & 0.15 & 0.48 & 0.64 & 0.79 & 0.001 & 0.92 & $<0.001$ & 0.03 & 0.003 \\
\hline
\end{tabular}

${ }^{1}$ The average EBV of the population is 100 and the genetic SD is 4.47 units for all the health traits and persistency. Longevity is expressed in days and has an average of 0 and a genetic SD of $270 \mathrm{~d}$. The $P$-value is based on a 2 -sided Student's $t$-test with 2 samples assuming equal variance.

${ }^{2} \mathrm{CaI}=$ calving interval; $\mathrm{FL}=$ interval from first to last insemination; $\mathrm{DS}=$ direct survival at birth; MS $=$ maternal survival at birth; UDH $=$ udder health index; CLW = claw health index; KET = ketosis; LON = longevity.

genetic effects for resistance and tolerance to endemic diseases; mastitis and ketosis are both clear examples of endemic diseases. Therefore, it is expected that EBV for residual variance partly captures genetic variation in disease resilience. Beyond disease resilience, EBV for residual variance may capture variation in general resilience: the ability of animals to maintain performance during any environmental perturbation (Colditz and Hine, 2016). From ecology, it is also known that a resilient system shows a small variance, whereas a system with increased variance have a higher probability to move to another state (Holling, 1973; Scheffer et al., 2012). In a dairy cow context, this may mean that animals with a small variance in performance stay healthy, whereas cows with increased variance are at risk to get diseased or die. In dairy cattle breeding, the question is whether resilience has an economic value beyond the economic values of specific diseases and longevity. Resilience could be related to easy-to-manage or troublefree production. In a farm with AMS and other sensors, resilience may be related to the number of alerts per cow per lactation. Increasing resilience by breeding (i.e., cows with a lower variance) is expected to yield cows that have fewer alerts and are thus requesting less labor from the farmer. Therefore, the economic value of resilience may be related to saving labor costs per cow and, in addition, resilience may have a noneconomic value related to higher satisfaction by the farmer (i.e., fewer problems due to cows with troubles). Indeed, our results show the EBV for LnVar could help to select cows with less mastitis and ketosis that stay longer on the farm (Tables 6 and 7). This is the first study, to our knowledge, showing the potential of using the variance of longitudinal data recorded by AMS that can be used to breed healthy, long-lasting cows that may be more resilient and easy to manage.

\section{CONCLUSIONS}

We found that traits related to fluctuations in milk yield are heritable, with heritabilities ranging from 0.06 to 0.10 . Furthermore, we found low to moderate ge- netic correlations between these fluctuation traits and existing fertility, health, and longevity traits. The logtransformed variance of milk yield appeared to be the best predictor among the fluctuation traits to predict udder health, ketosis, and longevity. This study shows that variability in performance contains information about health and longevity of cows. This research is a stepping stone to develop an EBV for variability of milk yield to facilitate breeding resilient and easy-tomanage dairy cows.

\section{ACKNOWLEDGMENTS}

CRV (the Netherlands) is acknowledged for providing the data.

\section{REFERENCES}

Blanchard, P. J., R. W. Everett, and S. R. Searle. 1983. Estimation of genetic trends and correlations for Jersey cattle. J. Dairy Sci. 66:1947-1954. https://doi.org/10.3168/jds.S0022-0302(83)82033 $-5$.

Calo, L. L., R. E. McDowell, L. D. Van Vleck, and P. D. Miller. 1973. Genetic aspects of beef production among Holstein-Friesians pedigree selected for milk production. J. Anim. Sci. 37:676-682. https://doi.org/10.2527/jas1973.373676x.

Codrea, M. C., S. Hojsgaard, and N. C. Friggens. 2011. Differential smoothing of time-series measurements to identify disturbances in performance and quantify animal response characteristics: An example using milk yield profiles in dairy cows. J. Anim. Sci. 89:3089-3098. https://doi.org/10.2527/jas.2010-3753.

Colditz, I. G., and B. C. Hine. 2016. Resilience in farm animals: biology, management, breeding and implications for animal welfare. Anim. Prod. Sci. 56:1961-1983. https://doi.org/10.1071/an15297.

CRV. 2011. E-23 Vitality Index, Maternal Vitality Index. Accessed May 31, 2017. https://www.crv4all-international.com/wp-content/ uploads/2016/03/E-23-Livability.pdf.

CRV. 2012a. E-7 Breeding value estimation of milk production traits with test-day model. Accessed May 31, 2017. https://www .crv4all-international.com/wp-content/uploads/2016/03/E-7-milk -production.pdf.

CRV. 2012b. E-19 Breeding value for longevity. Accessed May 31, $2017 . \quad$ https://www.crv4all-international.com/wp-content/ uploads/2016/03/E-19-Longevity.pdf.

CRV. 2014. E-30 Claw health index. Accessed May 31, 2017. https:// www.crv4all-international.com/wp-content/uploads/2016/03/E -30-Claw-health.pdf.

CRV. 2015. E-17 Breeding Value Fertility. Accessed May 31, 2017. https://www.crv4all-international.com/wp-content/uploads/ 2016/03/E-17-Fertility.pdf. 
CRV. 2016. E-27 Breeding value udder health. Accessed May 31, 2017. https://www.crv4all-international.com/wp-content/uploads/ 2016/04/E_27_apr2016_eng.pdf.

Damgaard, L. H., L. Rydhmer, P. Lovendahl, and K. Grandison. 2003. Genetic parameters for within-litter variation in piglet birth weight and change in within-litter variation during suckling. J. Anim. Sci. 81:604-610. https://doi.org/10.2527/2003.813604x.

Doeschl-Wilson, A. B., B. Villanueva, and I. Kyriazakis. 2012. The first step towards genetic selection for host tolerance to infectious pathogens: Obtaining the tolerance phenotype through group estimates. Front. Genet. 3:265. https://doi.org/10.3389/fgene.2012 .00265 .

Falconer, D. S., and T. F. C. Mackay. 1996. Introduction to Quantitative Genetics. 4th ed. Pearson Education Limited, Essex, UK.

Gilmour, A. R., B. J. Gogel, B. R. Cullis, S. J. Welham, and R. Thompson. 2014. ASReml User Guide Release 4.0. VSN International Ltd., Hemel Hempstead, UK.

Häggman, J., and J. Juga. 2013. Genetic parameters for hoof disorders and feet and leg conformation traits in Finnish Holstein cows. J. Dairy Sci. 96:3319-3325. https://doi.org/10.3168/jds.2012-6334.

Heringstad, B., G. Klemetsdal, and J. Ruane. 2000. Selection for mastitis resistance in dairy cattle: a review with focus on the situation in the Nordic countries. Livest. Prod. Sci. 64:95-106. https://doi .org/10.1016/S0301-6226(99)00128-1.

Hill, W. G., and H. A. Mulder. 2010. Genetic analysis of environmental variation. Genet. Res. (Camb.) 92:381-395. https://doi.org/10 $.1017 /$ S0016672310000546.

Holling, C. S. 1973. Resilience and stability of ecological systems. Annu. Rev. Ecol. Syst. 4:1-23.

Kadarmideen, H. N., R. Thompson, and G. Simm. 2000. Linear and threshold model genetic parameters for disease, fertility and milk production in dairy cattle. Anim. Sci. 71:411-419. https://doi.org/ 10.1017/S1357729800055338.

Kapell, D., C. J. Ashworth, P. W. Knap, and R. Roehe. 2011. Genetic parameters for piglet survival, litter size and birth weight or its variation within litter in sire and dam lines using Bayesian analysis. Livest. Sci. 135:215-224. https://doi.org/10.1016/j.livsci.2010 .07 .005 .

Koeck, A., J. Jamrozik, F. S. Schenkel, R. K. Moore, D. M. Lefebvre, D. F. Kelton, and F. Miglior. 2014. Genetic analysis of milk betahydroxybutyrate and its association with fat-to-protein ratio, body condition score, clinical ketosis, and displaced abomasum in early first lactation of Canadian Holsteins. J. Dairy Sci. 97:7286-7292. https://doi.org/10.3168/jds.2014-8405.

Koeck, A., F. Miglior, J. Jamrozik, D. F. Kelton, and F. S. Schenker. 2013. Genetic associations of ketosis and displaced abomasum with milk production traits in early first lactation of Canadian Holsteins. J. Dairy Sci. 96:4688-4696. https://doi.org/10.3168/jds $.2012-6408$

Koeck, A., F. Miglior, D. F. Kelton, and F. S. Schenkel. 2012. Health recording in Canadian Holsteins: Data and genetic parameters. J. Dairy Sci. 95:4099-4108. https://doi.org/10.3168/jds.2011-5127.

Mulder, H. A., J. M. Herrero-Medrano, E. Sell-Kubiak, P. K. Mathur, and E. F. Knol. 2015. Statistical models to increase disease resilience and uniformity in animal production. Page 453 in Proc. EAAP, Warsaw, Poland. Wageningen Academic Publishers, Wageningen, the Netherlands.

Mulder, H. A., L. Rönnegård, W. F. Fikse, R. F. Veerkamp, and E. Strandberg. 2013. Estimation of genetic variance for macro- and micro-environmental sensitivity using double hierarchical generalized linear models. Genet. Sel. Evol. 45:23. https://doi.org/10 $.1186 / 1297-9686-45-23$
Mulder, H. A., J. Visscher, and J. Fablet. 2016. Estimating the purebred-crossbred genetic correlation for uniformity of eggshell color in laying hens. Genet. Sel. Evol. 48:39. https://doi.org/10.1186/ s12711-016-0212-2.

Onyiro, O. M., J. Offer, and S. Brotherstone. 2008. Risk factors and milk yield losses associated with lameness in Holstein-Friesian dairy cattle. Animal 2:1230-1237. https://doi.org/10.1017/ S1751731108002279.

Ott, R. L., and M. T. Longnecker. 2010. An Introduction to Statistical Methods and Data Analysis. 6th ed. Brooks/Cole, Belmont, CA.

Parker-Gaddis, K. L., J. B. Cole, J. S. Clay, and C. Maltecca. 2014 Genomic selection for producer-recorded health event data in US dairy cattle. J. Dairy Sci. 97:3190-3199. https://doi.org/10.3168/ jds.2013-7543.

Rajala-Schultz, P. J., Y. T. Grohn, and C. E. McCulloch. 1999. Effects of milk fever, ketosis, and lameness on milk yield in dairy cows. J. Dairy Sci. 82:288-294. https://doi.org/10.3168/jds.S0022 -0302(99)75235-5.

Rönnegård, L., M. Felleki, W. F. Fikse, H. A. Mulder, and E. Strandberg. 2013. Variance component and breeding value estimation for genetic heterogeneity of residual variance in Swedish Holstein dairy cattle. J. Dairy Sci. 96:2627-2636. https://doi.org/10.3168/ jds.2012-6198.

Rupp, R., and D. Boichard. 2003. Genetics of resistance to mastitis in dairy cattle. Vet. Res. 34:671-688. https://doi.org/10.1051/vetres: 2003020.

Scheffer, M., S. R. Carpenter, T. M. Lenton, J. Bascompte, W. Brock, V. Dakos, J. van de Koppel, I. A. van de Leemput, S. A. Levin, E. H. van Nes, M. Pascual, and J. Vandermeer. 2012. Anticipating Critical Transitions. Science 338:344-348. https://doi.org/10 .1126 /science.1225244.

Sell-Kubiak, E., P. Bijma, E. F. Knol, and H. A. Mulder. 2015. Comparison of methods to study uniformity of traits: Application to birth weight in in pigs. J. Anim. Sci. 93:900-911. https://doi.org/ 10.2527/jas.2014-8313.

van der Drift, S. G. A., K. J. E. van Hulzen, T. G. Teweldemedhn R. Jorritsma, M. Nielen, and H. C. M. Heuven. 2012. Genetic and nongenetic variation in plasma and milk beta-hydroxybutyrate and milk acetone concentrations of early-lactation dairy cows. J. Dairy Sci. 95:6781-6787. https://doi.org/10.3168/jds.2012-5640.

van der Linde, C., G. de Jong, E. P. C. Koenen, and H. Eding. 2010. Claw health index for Dutch dairy cattle based on claw trimming and conformation data. J. Dairy Sci. 93:4883-4891. https://doi .org/10.3168/jds.2010-3183.

van der Waaij, E. H., M. Holzhauer, E. Ellen, C. Kamphuis, and G. de Jong. 2005. Genetic parameters for claw disorders in Dutch dairy cattle and correlations with conformation traits. J. Dairy Sci. 88:3672-3678. https://doi.org/10.3168/jds.S0022-0302(05)73053 -8 .

Vandenplas, J., C. Bastin, N. Gengler, and H. A. Mulder. 2013. Genetic variance in micro-environmental sensitivity for milk and milk quality in Walloon Holstein cattle. J. Dairy Sci. 96:5977-5990. https://doi.org/10.3168/jds.2012-6521.

Vosman, J. J., G. De Jong, H. Eding, and H. Knijn. 2015. Genetic evaluation for ketosis in the Netherlands based on FTIR measurements. Interbull Bull. 49:1-5. https://journal.interbull.org/index .php/ib/article/viewFile/1365/1433.

Windig, J. J., M. P. L. Calus, and R. F. Veerkamp. 2005. Influence of herd environment on health and fertility and their relationship with milk production. J. Dairy Sci. 88:335-347. https://doi.org/10 .3168/jds.S0022-0302(05)72693-X. 